\title{
Videolaringoscopy Beyond Conventional Endotracheal Intubation in HEMS: A Real Flight Simulation
}

\section{Gianluca Facchetti}

National Medical School of Italian Alpine Rescue Corps, Milan, Italy

Aglaia Gyra ( $\square$ aglaiagyra@gmail.com )

ASL 4 Teramo: Azienda Sanitaria Locale 4 Teramo https://orcid.org/0000-0003-1866-8677

Chiara Angeletti

ASL 4 Teramo: Azienda Sanitaria Locale 4 Teramo

\section{Francesco Masedu}

University of L'Aquila Department of Clinical Medicine Public Health Life Sciences and Environment: Universita degli Studi dell'Aquila Dipartimento di Medicina Clinica Sanita Pubblica Scienze della Vita e dell'Ambiente

\section{Enrico Molineris}

National Medical School of Italian Alpin Rescue Corps, CNSAS, Milan, Italy

\section{Dario Franchi}

National Medical School of Italian Alpine Rescue Corps, CNSAS, Milan, Italy

Lorenzo Introzzi

National Medical School of Italian Alpine Rescue Corps, CNSAS, Milan, Italy

Matteo Lucchelli

National Medical School of Italian Alpine Rescue Corps, CNSAS, Milan, Italy

\section{Andrea Molesi}

National Medical School of Italian Alpine Rescue Corps, CNSAS, Milan, Italy

Franco Marinangeli

University of L'Aquila Department of Clinical Medicine Public Health Life Sciences and Environment: Universita degli Studi dell'Aquila Dipartimento di Medicina Clinica Sanita Pubblica Scienze della Vita e dell'Ambiente

\section{Valter Bucci†}

National Medical School of Italian Alpine Rescue Corps, CNSAS, Milan, Italy

\section{Original research}

Keywords: Intubation, HEMS, video-laringoscopy, frontal right-lateral sitting position 
Posted Date: September 30th, 2021

DOl: https://doi.org/10.21203/rs.3.rs-927116/v1

License: (c) (i) This work is licensed under a Creative Commons Attribution 4.0 International License. Read Full License 
Videolaringoscopy beyond conventional endotracheal intubation in HEMS: a real flight simulation

Facchetti $\mathrm{G}^{1}$, Gyra $\mathrm{A}^{2}$, Angeletti $\mathrm{C}^{2}$, Masedu $\mathrm{F}^{3}$, Molineris $\mathrm{E}^{1}$, Franchi $\mathrm{D}^{1}$, Introzzi $\mathrm{L}^{1}$, Lucchelli $\mathrm{M}^{1}$, Molesi $\mathrm{A}^{1}$, Marinangeli $\mathrm{F}^{4}$, Bucci $\mathrm{V}^{1 \dagger}$

1. National Medical School of Italian Alpine Rescue Corps, CNSAS, Milan, Italy.

2. Operative Unit of Anaesthesiology, Intensive Care and Pain Medicine, Civil Hospital G. Mazzini, Teramo, Italy

3. Department of Biotechnological and Applied Clinical Sciences, Section of Clinical Epidemiology and Environmental Medicine, University of L'Aquila, L'Aquila, Italy.

4. Department of Life Health and Environmental Sciences, Anaesthesiology and Pain Medicine, University of L'Aquila, L'Aquila, Italy

Corresponding Author: Aglaia Gyra M.D.

Affiliation: Operative Unit of Anaesthesiology, Intensive Care and Pain Medicine, Civil Hospital G. Mazzini, Teramo, Italy

Address: Piazza Italia 1, Teramo 64100

Mail: aglaiagyra@gmail.com

TEL: 0039-3208606779 


\begin{abstract}
Background: In pre-hospital setting, airway management may be required during hems transportation, when patients deteriorate while en route. Our primary objective was to assess whether in-flight indirect laryngoscopy in frontal right-lateral sitting position (FP) of the operator, is such effective as traditional indirect laryngoscopy methods onshore, considering, also, the association of success rate with influencing flight factors (flying, devices, position, comfort VAS, Lumen VAS).
\end{abstract}

Methods: This observational prospective study, aimed to estimate the success rate of in-flight FP indirect laryngoscopy intubation. The study conducted on an AW 169 helicopter (CLeonardo Company, Italy) equipped with transversal stretcher, during steady flight. Indirect laryngoscopy devices (GlideScope Ranger and AirTraq) were used on a HAL® manikin (Accurate, Gaumard® Scientific Company,Inc) with cervical collar.

Results: All of the FP intubations with both devices were successful after two attempts maximum. The mean time for intubation was slightly greater using the Glide Scope Ranger

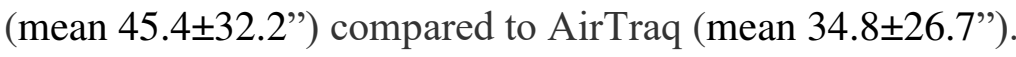

Conclusions : Both AirTraq and GlideScope Ranger used in in-flight FP guarantee a comparable level of effectiveness to traditional onshore method with $100 \%$ successful rate of intubation, Airtraq seems not been affected by Lumen, indicating that in this specific contest it guarantees a high visualisation regardless the light conditions. On the other hand, the FP results effective using AirTraq in both conditions.

\title{
Keywords
}

Intubation, HEMS, video-laringoscopy, frontal right-lateral sitting position. 


\section{Background}

Critically ill or injured patients need to be immediately identified, properly managed and rapidly transported to definitive care. According to the algorithm of Pre-hospital Trauma Life Support, the A-B-C-D-E approach is fundamental during patient's assessment [1]. All patients undergoing medical evacuation by helicopter may require, in the meanwhile, emergent intubation. Improved training and expertise have enabled emergency medical personnel to provide advanced levels of care at the scene of trauma. Advanced techniques, in the hostile environment or whenever transport times are prolonged, should be guaranteed both, on field and in the helicopter, in order to assure a favourable outcome. Successful emergency airway management is an essential component of the modern practice of rescue medicine and it has guaranteed during both day and night missions.

Airway management may be required during medical evacuation in a helicopter when patients deteriorate en-route. Direct laryngoscopy can be challenging, even for welltrained, experienced personnel. The repercussions for unsuccessful or prolonged attempts at intubation can be dramatic, in case of difficult airways, are not limited to the operating room context but can be also apply to the pre-hospital setting of helicopter emergency medical service (HEMS) [2]. In addition, laryngoscopes positioning at the head of the patient during in-cabin manoeuvres may not be always possible, making it difficult to perform traditional, direct laryngoscopy.

In the last years, the use of a video laryngoscope as the primary method of intubation has been shown in many cases to be at least as good as, and often more successful than, direct laryngoscopy [3].

Owing to the development of medical technology, there are an increasing number of video laryngoscopes and other devices facilitating endotracheal intubation in several airway scenarios. Each of these devices may bring benefits by increasing the in-flight intubation 
effectiveness, as well as shortening the procedure, provided that, the healthcare personnel performing intubation is familiar with the use of the device.

Most intubations by HEMS crews occur before loading the patient into the helicopter. However, some in-flight intubations are necessary owing to unforeseen circumstances, such as patient deterioration in-flight or accidental dislodgement of an endotracheal tube. Should an in-flight intubation be necessary, direct laryngoscopy becomes more difficult by space and mobility limitations. Simply put, in the cramped quarters of a helicopter, it can be difficult to obtain the proper line of sight needed to right place the endotracheal tube using direct laryngoscopy [4].

While the skill of tracheal intubation via direct laryngoscopy taught to many healthcare professionals, it is a difficult skill to acquire and maintain [5]. Serious consequences may result from a poorly performed intubation attempt. The rate of airway-related complications correlates with an increased number of intubation attempts. The increased number of laryngoscopy attempts increases the incidence of hypoxemia, aspiration, bradycardia, and cardiac arrest [6] (Thus, successful intubation occurring on the initial attempt is imperative.

Several studies compared direct laryngoscopy using a Macintosh blade with Videoassisted intubation devices and found a more rapid acquisition of skills and faster and more consistent intubation in difficult scenarios and less theoretical dental trauma (in intubations performed on a manikin) [7-9,5].

First-time users of video assisted intubation devices have an improved view of the glottis during difficult airway situations compared to direct laryngoscopy [10]. The skill of novice laryngoscopists diminishes rapidly over a period of several months without intervening practice [11]. Alternatively, a laryngoscopy approach is available to perform endotracheal 
intubation from a ventral position while directly facing the victim. This technique repeatedly reported, but systematic data on the usefulness of this approach are scarce.

In recent years, alternative intubation techniques, including indirect optical laryngoscopy and video laryngoscopy, increasingly used to facilitate tracheal intubation [12]. Because of this, such techniques may also be useful in the context of face-to-face intubations.

Particularly in HEMS, in-flight endotracheal intubation is impossible to take place in case of transversal stretchers, because of the limited space of the cabin which does not allow to the Anaesthetist to get positioned behind the head of the patient, in order to perform traditional direct laryngoscopy. Therefore, we hypothesized that a new point of view may facilitate in-flight, in-cabin endotracheal intubation in helicopters of big dimensions, furnished with winch and transversal stretcher. Thus, we supposed that the frontal-rightlateral position of the operator by using indirect video-laryngoscopy is such efficient as the traditional direct laryngoscopy.

This research is focused on proving that in-flight, in-cabin, indirect laryngoscopy by AIRTRAQ $^{\text {TM }}$ or GLIDESCOPE ${ }^{\circledR}$ RANGER in frontal-right-lateral position, is able to guarantee airway management.

\section{Materials and Methods}

We designed this observational study in prospectively collected data, comparing intubating conditions in two different settings: in-flight versus onshore one. The objective of this study was to assess whether in-flight indirect laryngoscopy in frontal right-lateral sitting position of the operator, was such effective as traditional position indirect laryngoscopy methods onshore. 
Secondly, we compared the two devices used for indirect laryngoscopy: Airtraq ${ }^{\circledR}$ versus Glade Ranger®, evaluating the intubation times, the lighting conditions and the operator's comfort during the manoeuvre, in the cabin, during the flight and on the ground setting. The National Corps of Mountain and Speleological Rescue (CNSAS, Italy) and the National Medical School of the National Corps of Mountain and Speleological Rescue supported the research. Leonardo Company (Aerospace, Defence, Security) provided 48 hours of availability in Vergiate heliport in order to perform in-flight manoeuvres on an AW 169 helicopter.

Participants classified based on previous experience with laryngoscopy and included residents in anaesthesiology, anaesthesiology attending physicians, and emergency medicine physicians. The convenience sample included a Novice group (3 participants) and an Expert group (7 participants) who self-identified as having at least 30 lifetime intubations in emergency in hostile environment.

The study conducted in the patient cabin of medevac-configured AW169 helicopter (CLeonardo Company, Italy). A Mallampati I, first grade Cormack-Lehane trauma adult HALL® S 1000di Accurate-Gaumard mannequin, with adult cervical collar Laerdal Stifneck®, was positioned supine on the secured installed helicopter stretcher.

In-flight intubations performed at the period included between the take off and the landing. Thus, the first four minutes (take off) and the last four minutes (landing) were excluded from any manoeuvre. During the flight, the pilot set a steady flight at 2000 feet with a constant velocity of 90 knots ad a maximum grade of veer of 8 grades. All in-flight intubations were performed with frontal approach to the mannequin by the belted operator in a right-lateral sitting position respect to the head of the mannequin. Control group intubations were performed onshore, while helicopter was stationary on the ground, engines off, with the cage open, in order to permit indirect laryngoscopy with devices in both approaches: the traditional one, behind of the head of the patient and the frontal-right-lateral one. 


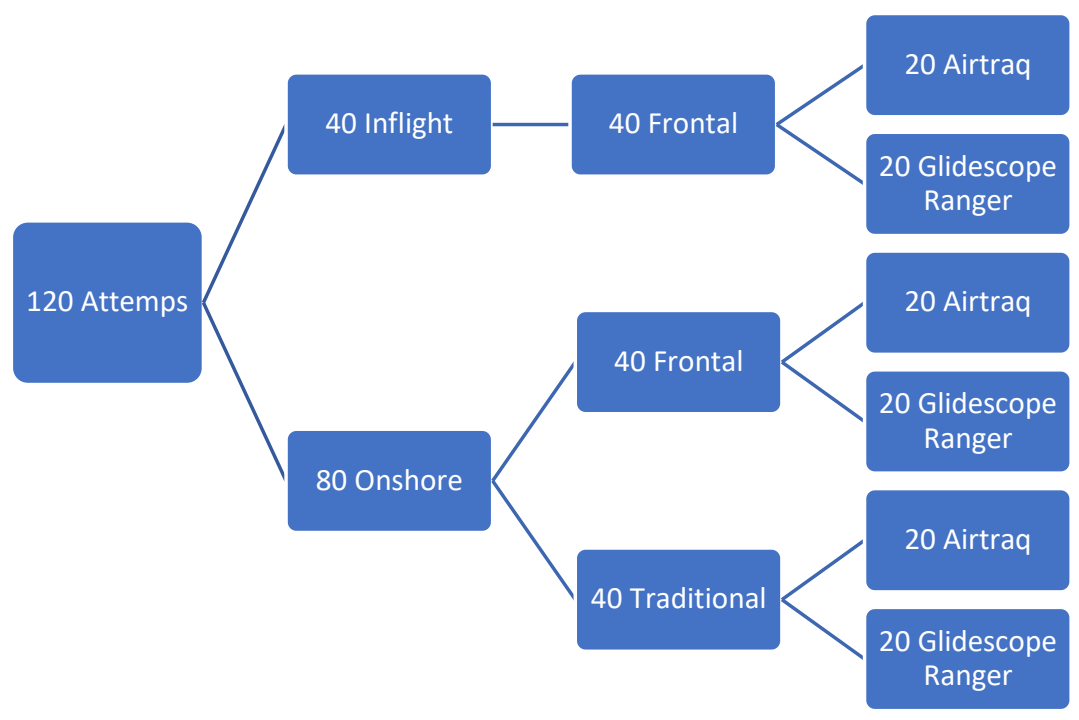

Design of the study: sequential attempts.

Each participant used the two devices in a randomized order and executed a total number of 12 intubations:

a) 4 In-flight intubations in frontal-right-lateral position (two for each device: Airtraq ${ }^{\circledR}$ and Glidescope ${ }^{\circledR}$ Ranger);

b) 4 Onshore frontal-right-lateral position intubations while helicopter was stationary on the ground in the hangar (two for each device);

c) 4 Onshore traditional position intubations with the operator behind the head of the patient (two for each device), while helicopter was stationary on the ground, in the hangar. (Control group intubations).

Intubation attempts conducted independently, without assistance. Elapsed time recording commenced when the device passed through the lips of the manikin and stopped when the laryngoscope blade removed from the manikin's lips, after the final completed intubation attempt. Successful intubation defined by investigator visual verification of the lung expansion. No specific performed training on the devices prior to use in the study. Therefore, participants may have used a different device at their home hospital. 
Each participant permitted up to three attempts with a maximum time allotment of 4 min per attempt to successfully intubated manikin attempts. Subjects had a total time of $12 \mathrm{~min}$ per device to achieve tracheal intubation and the opportunity to reassess their technique and make a new attempt. The time to successful intubation was recorded. Permitted optimization manoeuvres verbalized to each participant prior to their attempts at intubation and included external laryngeal manipulation and simple manipulation of the manikin's head.

Immediately following each intubation attempt, participants gave a brief survey in order to express their personal perception of comfort during each attempt. After the use of each Intubation Device subjects reported a Comfort VAS Scale consisting of a subjective "ease of use" evaluation using a 10-point Comfort VAS, ranging $1=$ "Extremely easy" to $10=$ "Extremely difficult".

The amount of light emitted during every intubation detected in order to evaluate its affection on the success rate. LMT® Pocket-LUX 2 calibrated the illumination reflecting to devices. The different lumen registrations during every attempt were distributed in 5 different Lumen classes: 1-2000 lumen= Lumen Class 1, 2001-4000 lumen=Lumen Class 2, 4001-6000 lumen= Lumen Class 3, 6001-8000 lumen= Lumen Class 4, 8001-10000 lumen= Lumen Class 5. The Lumen measurements taken at distance of $20 \mathrm{cms}$, on the left side of the mannequin. All phases of the experiment registered by full-hd camera in order to evaluate and discuss collected data after the debriefing.

\section{$\underline{\text { Statistical analysis }}$}

Success rate was the primary outcome on which sample size has calculated. A previous study reported a success rate of $88 \%$ for inverse intubation of the manikin with Airtraq ${ }^{\circledR}$ [13].

We consider and presume that a useful alternative technique should have a success rate of more than $90 \%$ (ideally approaching $100 \%$ ). Hence, we powered our study to detect a clinically 
meaningful difference in success rate of more than $15 \%$. With a targeted power of $80 \%$ and a two-tailed $\alpha$ level of 0.05 ; this requires a sample size of 20 per group.

The primary endpoint was successful establishment of endotracheal airway intubation. Normal distribution of linear data has been proven by Kolmogorov-Smirnov. Mann-Whitney U test (two-tailed), Q-square test, T-test and were used to detect significant differences among groups investigating as appropriate.

The association of success rate with influencing factors (flying, devices, position, comfort VAS, Lumen VAS) was assessed using Logistic Regression Analysis and Pearson correlation. Linear regression analysis has been used for evaluating the association of intubation success rate with potential influencing factors. A p-value of 0.05 ( $<<0.05$ was considered statistically significant) was deemed to be statistically significant throughout the study.

\section{Results}

A total number of 120 intubations were performed; $n=80(60 \%)$ intubations successfully executed in the helicopter while stationary on the ground. A total number of $n=40(40 \%)$ intubations were performed during steady flight in frontal-right-lateral position.

Among those 120 intubations $n=80(60 \%)$ have been performed in frontal position and $n=40$ $(40 \%)$ in the traditional, rear one. Regarding the device use, $n=60(50 \%)$ manoeuvres were executed with the Aitraq and $n=60(50 \%)$ with the GRS.

\section{Success Rate}

Successful intubation, at the first attempt obtained in 114 cases $(95 \%)$ while in $5 \%$ at the second attempt.

There is no significant difference in the success rate of intubation between the devices (AT Group $1.03 \pm 0.02$ vs GRS Group 1.06 $\pm 0.03 ;-0.112-0.045$ [95\% CI], p=0.406). 
It results that there is no influence to the success rate of intubations due to the position: FRLPos $n=80$ vs TRADPos $n=40$ (FRL Pos1.03 \pm 0.02 vs TRAD Pos $1.075 \pm 0.42 ;-0.12-0.046$ [95\% CI], $\mathrm{p}=0.3785)$. Similarly, in-flight intubation $(\mathrm{n}=40)$ does not affect the success rate compared to the onshore one $(\mathrm{n}=80)$, there is no significant difference in the success rate of intubations (INFLIGHT 1.075 \pm 0.04 vs ONSHORE 1.03 $\pm 0.02 ;-0.12-0.04[95 \% \mathrm{CI}], \mathrm{p}=0.376$ ).

\section{Endotracheal tube insertion time}

The comparison of the required time for successful intubation between the devices, demonstrates a higher performance in terms of time execution and success with the procedure performed with AT than GRS (AT Group n=60 mean 34.8 \pm 26.7 , median 22 sec vs GSR Group $\mathrm{n}=60$, mean $45.4 \pm 32.2$, median $35.2 \mathrm{sec} ; \mathrm{p}=0.0003$ ). Nevertheless, the comparison of time needed for successful intubation by both devices during in flight manoeuvres (right lateral frontal position) compared to the one executed onshore by traditional rear position (control group), shows that there is no statistically significant difference $(\mathrm{p}=0.101 ; \mathrm{p}=0.062)$.

\section{Subjective comfort rating and Lumen class}

Regarding the difference in Comfort VAS score, expressed as a categorical scale from 1 to 10, by the operator, the comparison showed a statistical significance, with better comfort VAS expressed when intubating with Airtraq (AT Group $\mathrm{n}=60$ mean $1.75 \pm 0.9$, median 2 vs GSR Group $\mathrm{n}=60$, mean 4.5 \pm 2.6 , median $4 ; \mathrm{p}=0.00001$ ).

Similarly, during inflight simulation in frontal-right-lateral position the best comfort has been expressed when using the Airtraq $(\mathrm{p}=0.001)$.

The Lumen Class difference during the manoeuvres with both devices is not statistically significant $(\mathrm{p}=0.799)$, creating this way homogeneous conditions that permit to obtain a reliable comparison between the devices. 
Linear regression analysis and correlation indicates how the intubation success rate is influenced by the ambient conditions of luminosity (lumen class) $(\mathrm{p}=0.018)$, the success at the first attempt and the perception of well-being of the operator expressed through comfort VAS $(\mathrm{p}=0.0001)$ indicates how in general ambient light seems to affect the maneuver.

The correlation between Comfort VAS and Lumen class is statistically significant (rs= 0.208, P2-tailed=0.02216, Pearson correlation)

\section{Discussion}

Intubation of the normal airway in a well-lit environment may be challenging for the inexperienced or infrequent laryngoscopist. This challenge is multiplied in prehospital or hostile environment where the medical doctor may need to intubate a patient's airway under suboptimal conditions, such as low or extremely high light, on upper-level stanchions or the floor of the aircraft, or when providers cannot easily place themselves at the head of the patient due to space restrictions of the aircraft. The use of a Videolaryingoscope Indirect Intubation in this type of environment likely increases the first-pass success rate because it provides a clear visualization of the glottis opening for providers, who may or may not be seasoned or frequent laryngoscopists. Videolaryngoscopy provides several advantages. First, it may make an impossible intubation possible (or even easier) because accessing the airway with the laryngoscope and a hand may be far easier than positioning the intubator's head and torso into a position whereby a direct line-of-sight of the airway is achieved. Videolaryngoscopy allows a much better view of the pharyngeal and laryngeal structures than classic laryngoscopy [14])

As we described, endotracheal intubation may occur during Helicopter Emergency Medical Service [15]. This can especially be challenging with entrapped casualties when access to the patient is restricted. In such situations, prehospital emergency personnel will 
usually administer oxygen and keep the airway open using basic airway techniques or supraglottic airway devices until the patient has been extricated [16].

However, if rapid extrication is not possible and when life-threatening airway obstruction and severe hypoxia persist, the advanced life support (ALS) provider may-in rare instances - be forced to attempt securing the airway with an endotracheal tube while the patient is still entrapped. Herein, conventional laryngoscopy is often not possible due to limited access to the head, and some ALS providers advocate the use of a primary surgical technique under such circumstances. Alternatively, a laryngoscopic approach is available to perform endotracheal intubation from a ventral position ("sitting" patient position) while directly facing the victim. This technique has repeatedly been reported, but systematic data on the usefulness of this approach are scarce, especially in a confined environment such as the cabin of an air ambulance [17-18, 13].

\section{Success Rate}

In an environment, such confined as a helicopter, the videolaryngoscopy advantage of not requiring line-of-sight to intubate, might make intubation possible where it would have been impossible using direct laryngoscopy. Video laryngoscopes were developed to provide improved visualization and might help in these situations. Videolaryngoscopy also provides an added benefit of providing a method for quality improvement on intubation methods and performance that direct laryngoscopy cannot provide [19].

Brown et al. retrospectively reviewed intubation success rates from an 89 rotorcraft air medical system from January 1, 2007, through December 31, 2009 and concluded that the Airtraq device achieves success rates better than or at least comparable with other air medical direct laryngoscopy success rates when using mannequin [20]. 
Our study clearly demonstrates that there is a $100 \%$ successful intubation with both devices. Success Rate was not influence by the kind of the device, the position and the inflight or onshore condition. Kronhall et al. in their study, registered a $100 \%$ in-cabin intubation success rate, too, in combination with how participants found in-cabin intubation conditions equal to or better than standard conditions [15]. This means that now and on, the scientific and the emergency medicine world should consider seriously indirect video-laryngoscopy as a valid concept to face since the first attempt, airway management en-route. Wider use of videolaryngoscopes in HEMS would increase the number of first-attempt successful endotracheal intubation in difficult airway scenarios.

\section{$\underline{\text { Endotracheal tube insertion time }}$}

The expectation of modern HEMS was to perform advanced measures, including intubation without unnecessarily delaying hospital intervention. With our study, adequate time of inflight right-lateral-frontal successful intubation it was demonstrated, with a slightly higher performance in terms of time obtained by Airtraq than Glidescope Ranger. An absolute record time of successful frontal intubation (11'"39 seconds) was register by using Airtraq. Prolonged scene times may increase mortality in certain critical injuries and illnesses because it delays hospital treatment [21-22]. With specialist HEMS, the benefits of early intervention are thought to outweigh the detrimental effects of prolonged scene times [23-24].

\section{Subjective Comfort VAS rating and Lumen Class}

The Lumen Class homogeneity resulted by the accurate lumen registration during our study, permitted a reliable interpretation of collected data, for what concerns both devices' efficacy. Light emission is a remarkable concern when in-flight intubation, is operated during several favourable or forbidding conditions. Wallace et al. analysed light emission during endotracheal intubation in a high-fidelity patient simulator lab [25]. Even if in this study, light emission 
evaluation focused in avoiding ground-based observer to localize the aircraft in combat zones and avoid fire; our investigation is the unique underling efficient performance of endotracheal intubation associated to light emission.

Regarding our study, even both devices have a $100 \%$ successful rate of intubation, Airtraq seems not to be affect by Lumen, indicating that in this specific contest it guarantees a high visualisation regardless the light conditions.

Our findings suggest that in-cabin; en-route intubation can be performed, bypassing actual problems like space, stretcher position, and medical doctor seat thanks to videolaryngoscopic vision.

Use of video-laryngoscopy in prehospital settings will increase as within the next years, a further development of video-laryngoscopy is expected. We believe that video-laryngoscopy may facilitate practical execution of in-flight intubation guaranteeing a more optimal airway management under challenging conditions in an aeromedical aircraft. We are hopeful that further investigation of in-flight video-laryngoscopy, advances in technology and development of video-laryngoscopy management protocols will improve management of patient requiring intubation in helicopters.

\section{Study limitations}

As a pilot study, our experimentation remains a simulated intubation on a manikin with specific intubation parameters. The implications of our results on real-world situations are difficult to establish. Intubating conditions may differ from human trauma patients, for example, due to absence of facial trauma and bleeding. Therefore, we cannot exclude that, different results would be observe if blood or vomitus was present in the oral cavity, pharynx or airway. For example, a previous randomized controlled trial about prehospital use of the 
Airtraq device reports a success rate of only $47 \%$, identifying impaired sight due to blood or vomitus as one of the reasons of low success rate. (89) Future in-flight studies needed in order to assess the performance and safety of such technique in human patients.

A total number of 40 in-flight frontal-right-lateral intubations represents a small sample size that constrained the ability to generalize the results. But, even if it still remains a limited number for deep statistical elaboration, it did not affect significantly the statistical analysis.

The whole experience has been performed during daytime flight. It would be interesting extend data collection during night flight in order to have a global visualisation and analysis of collected data.

On the other hand, as there is a remarkable lack of in-flight intubation studies in literature, there is no possibility for further comparison of our results with previous data. Anyway, this may be a valid initial path, as our results for the first time in literature, demonstrate that intubating in frontal position during flight is possible.

In addition, may have affected comfort perception, during all in-flight frontal-right-lateral position intubations performed while the medical doctor belted in order to respect all aeronautical safety rules during flight.

\section{Conclusions}

There are definitively few studies in literature, considering in-cabin intubation performed by physicians working in civilian prehospital care. Similar in-cabin (but not in-flight) intubation data have been previously documented only in a military context, but, on much larger helicopters as a means of facilitating rapid evacuation under fire and as retrospectively collected data. 
Our findings reproduced, in an operational environment, in a steady flight, including this way potential critical environment conditions such as vibration, movement, noise and light exposure during the flight. This created a reliable simulation of in-flight intubation able to represent real conditions during the experimentation.

Nowadays it remains a unique in-cabin, in-flight study performed en-route and this definitively determines both the novelty idea and realization. We surely believe that innovation and practical investment in scientific research is the first path for results that could determine future medical management in HEMS. 


\section{Ethics approval and consent to participate}

The University of L'Aquila Local Review Board reviewed this study and classified it as exempt from full review because it did not meet its requirements for research involving human subjects as it was executed on a mannequin.

\section{Consent for publication}

Consent for publication of photos/videos was obtained from both the official photographer Mr. Pierluigi Facchetti and the official video reporter Mr. Diodato Salvatore. They both understood that the text and any pictures or videos eventually published in the article will be freely available on the internet and may be seen by the general public. The pictures, videos and text may also appear on other websites or in print, may be translated into other languages or used for commercial purposes. They have both been offered the opportunity to read the manuscript

\section{Availability of data and materials}

The datasets used and/or analysed during the current study are available from the corresponding author on reasonable request.

\section{Competing interests}

The Authors declare that they have no competing interests. The Authors have no relevant affiliations or financial involvement with any organization or entity with a financial interest in or financial conflict with the subject matter or materials discussed in the manuscript.

\section{Funding}

This research was supported by the National Corps of Mountain and Speleological Rescue (CNSAS, Italy,) and the National Medical School of the National Corps of Mountain and Speleological Rescue. Leonardo Company (Aerospace, Defence, Security) provided 48 hours of availability in Vergiate heliport in order to perform in-flight manoeuvres on an AW 169 helicopter. 


\section{Authors' contributions}

FG conception and design of the work, acquisition of data, revision; GA conception and design of the work, analysis and interpretation of data, bibliography, writing manuscript; AC analysis and interpretation of data, bibliography research, revision; $\mathrm{MF}$ analysis of data; $\mathrm{ME}$ bibliography research; FD bibliography research; IL bibliography research; LM bibliography research; MA revision; MF revision; $\mathrm{BV}^{\dagger}$ acquisition of data. All authors read and approved the final manuscript.

\section{Acknowledgements}

We acknowledge the contribution during the execution of the experimentation of the guests: Del Romano Mauro, De Paolis Valeria, Parisse Tiziana, Diodato Salvatore, Facchetti Pierluigi and the support of the Leonardo attendees: Tonini Luca, Bubbico Domenico, Raddi Francesca, Marolla Alberto, the pilot commander Missarino Piero, Cattaneo Andrea, Sacco Carlo, Cereda Claudio. Authors obtained permission to acknowledge from all those mentioned in this section.

Saving a life during a Helicopter Emergency Medical Service is the main target of all the rescuers that risk their own lives during every mission. On $24^{\text {th }}$ January 2017 a brilliant medical doctor, member of our scientific medical team, Dr. Valter Bucci, lost his life at the EC-KJT helicopter crash, during a HEMS transportation at Campo Felice, Italy.

Later, Engineer Luca Tonini, prematurely passed away on $8^{\text {th }}$ July 2019 . He has been the first to embrace our idea and thanks to his intuition invented the new concept of "flying hospital" that has become the target of our air medical mission in future.

This study is entirely dedicated to both of them, with the hope their memory will live forever. Rest in peace. 


\section{References}

1. David Häske, Stefan K Beckers, Marzellus Hofmann; Subjective safety and selfconfidence in prehospital trauma care and learning progress after trauma-courses: part of the prospective longitudinal mixed-methods EPPTC-trial; Scand J Trauma Resusc Emerg Med. 2017 Aug 14;25(1):79.

2. Caplan RA, Posner KL, WardRJ, et al. Adverse respiratory events in anesthesia: a closed claims analysis. Anaesthesiology 1990;72:828Y833.

3. Mosier JM, Law JA., Airway management in the critically ill. Intensive Care Med. 2014 May;40(5):727-9.

4. Thomas SH, Farkas A, Wedel SK. Cabin configuration and prolonged oral endotracheal intubation in the AS365N2 Dauphin EMS helicopter. Air Med J $1996 ; 15: 65 Y 68$

5. Maharaj CH, Costello JF, Higgins BD, et al. Learning and performance of tracheal intubation by novice personnel: a comparison of the Airtraq and Macintosh laryngoscope. Anaesthesia. 2006;61(7):671-7.

6. Mort TC. Emergency tracheal intubation: complications associated with repeated laryngoscopic attempts. AnesthAnalg. 2004;99(2):607-13.

7. Berg B, Walker RA, Murray WB, et al. Airway intubation in a helicopter cabin: video vs. direct laryngoscopy in manikins. Aviat Space Environ Med. 2009;80(9):820-3.

8. Wang PK, Huang CC, Lee Y, et al. Comparison of 3 video laryngoscopes with the Macintosh in a manikin with easy and difficult simulated airways. Am J Emerg Med. 2013;31(2):330-8. 15 .

9. Jungbauer A, Schumann M, Brunkhorst V, et al. Expected difficult tracheal intubation: a prospective 
comparison of direct laryngoscopy and video laryngoscopy in 200 patients. $\mathrm{Br} \mathbf{J}$ Anaesth. 2009; 102(4):546-50.

10. Boedeker BH, Barak-Bernhagen MA, Boedeker KA, et al. Intubation success rates and perceived user satisfaction using the video laryngoscope to train deploying far forward combat medical personnel. Stud Health Technol Inform. 2011;163:77-9.

11. Maharaj CH, Costello J, Higgins BD, et al. Retention of tracheal intubation skills by novice personnel: a comparison of the Airtraq and Macintosh laryngoscopes. Anaesthesia. 2007;62(3):272-8.

12. S R Lewis 1, A R Butler 1, J Parker; Videolaryngoscopy versus direct laryngoscopy for adult patients requiring tracheal intubation: a Cochrane Systematic Review; Br J Anaesth. 2017 Sep 1;119(3):369-383.

13. Schober P, Krage R, van Groeningen D et al. Inverse intubation in entrapped trauma casualties: a simulator based, randomised cross-over comparison of direct, indirect and video laryngoscopy. Emerg Med J. 2014 Dec;31(12):959-63.

14. Van Zundert A, Pieters B, Doerges V et al Videolaryngoscopy allows a better view of the pharynx and the larynx than classic laryngoscopy. Br J Anaesth 2012; 109:1014-5.

15. Daniel Kornhall, Fredrik Hellikson, Robert Näslund; A Protocol for Helicopter In-Cabin Intubation; Air Med J. 2018 Sep;37(5):306-311.

16. PE Jacobs and A Grabinsky; Advances in prehospital airway management; Int $\mathbf{J}$ Crit Illn Inj Sci. 2014 Jan-Mar; 4(1): 57-64.

17. Van Zundert TC, Van Zundert AA. Tracheal intubation of patients in non-standard positions requires training. Minerva Anestesiol. 2013 Jun;79(6):679-82. 
18. Komasawa N, Ueki R, Itani M, Nomura H, Nishi SI, Kaminoh Y. Evaluation of tracheal intubation in several positions by the Pentax-AWS Airway Scope: a manikin study. J Anesth. 2010 Dec;24(6):908-12.

19. Carlson JN, Quintero J, Guyette FX, et al. Variables associated with successful intubation attempts using video laryngoscopy: a preliminary report in a helicopter emergency medical service. Prehosp Emerg Care 2012;16:293Y29.

20. Brown CA, Cox K, Hurwitz S et al. 4,871 Emergency airway encounters by air medical providers: a report of the air transport emergency airway management (NEAR VI: "A-TEAM") project. West J Emerg Med. 2014 Mar;15(2):188-93.

21. McCoy CE, Menchine M, Sampson S, et al . Emergency medical services out-ofhospital scene and transport times and their association with mortality in trauma patients presenting to an urban level I trauma center. Ann Emerg Med. 2013;61:167174.

22. HarmsenAM, GiannakopoulosGF, MoerbeekPR, et al. FW.The influence of prehospital time on trauma patients outcome: a systematic review. Injury. 2015;46:602-609.

23. Brown JB, Rosengart MR, Forsythe RM et al. Not all prehospital time is equal: influence of scene time on mortality. J Trauma Acute Care Surg. 2016;81:93-100.

24. Pakkanen T, Kämäräinen A, Huhtala $\mathrm{H}$ et al. Physician-staffed helicopter emergency medical service has a beneficial impact on the incidence of prehospital hypoxia and secured airways on patients with severe traumatic brain injury. Scand J Trauma Resusc Emerg Med. 2017 Sep 15;25(1):94.

25. Matthew C. Wallace, SSgt Tyler Britton, Robbie Meek et al. Comparison of five video-assisted intubation devices by novice and expert laryngoscopists for use in the aeromedical evacuation environment. Mil Med Res. 2017; 4: 20. 